\title{
Communication \\ Near- to Far-Field Coupling of Evanescent Waves by Glass Microspheres
}

\author{
Rayenne Boudoukha ${ }^{1,2}{ }^{\infty}$, Stephane Perrin ${ }^{1, *}{ }^{-1}$, Assia Demagh ${ }^{2}$, Paul Montgomery ${ }^{1}$, Nacer-Eddine Demagh ${ }^{2}$ \\ and Sylvain Lecler ${ }^{1}$ (D) \\ 1 ICube Research Institute, CNRS-INSA Strasbourg-University of Strasbourg, 67412 Illkirch, France; \\ rayenne.boudoukha@etu.unistra.fr (R.B.); paul.montgomery@unistra.fr (P.M.); \\ sylvain.lecler@insa-strasbourg.fr (S.L.) \\ 2 Applied Optics Laboratory, Institute of Optics and Precision Mechanics, Ferhat Abbas University Setif1, \\ 19000 Setif, Algeria; assiademagh@univ-setif.dz (A.D.); ndemagh@univ-setif.dz (N.-E.D.) \\ * Correspondence: stephane.perrin@unistra.fr
}

\section{check for}

updates

Citation: Boudoukha, R.; Perrin, S.; Demagh, A.; Montgomery, P.; Demagh, N.-E.; Lecler, S. Near- to Far-Field Coupling of Evanescent Waves by Glass Microspheres. Photonics 2021, 8, 73. https:// doi.org/10.3390/photonics8030073

Received: 5 February 2021

Accepted: 4 March 2021

Published: 6 March 2021

Publisher's Note: MDPI stays neutral with regard to jurisdictional claims in published maps and institutional affiliations.

Copyright: (c) 2021 by the authors. Licensee MDPI, Basel, Switzerland. This article is an open access article distributed under the terms and conditions of the Creative Commons Attribution (CC BY) license (https:// creativecommons.org/licenses/by/ $4.0 /)$.

\begin{abstract}
Through rigorous electromagnetic simulations, the natural coupling of high-spatialfrequency evanescent waves from the near field to the far field by dielectric microspheres is studied in air. The generation of whispering gallery modes inside the microspheres is shown independently of any resonance. In addition, the conversion mechanism of these evanescent waves into propagating waves is analysed. This latter point leads to key information that allows a better physical understanding of the super-resolution phenomenon in microsphere-assisted microscopy where sub-diffraction-limit revolving power is achieved.
\end{abstract}

Keywords: microsphere; evanescent waves; propagation; whispering gallery modes; super-resolution

\section{Introduction}

In optical imaging, the spatial distribution of an object leads to a spatial modulation of the reflected (or transmitted) light. According to the diffraction theory of light, only a part of the electromagnetic waves having spatial frequencies smaller than $2 / \lambda$ ( $\lambda$, the wavelength of light) in air appears to be propagative and, thus, can be collected by an optical system. The high-spatial-frequency modulations remain localised around the object surface and are evanescent. With the aim to observe more and more details, various optical techniques have been developed in order to overcome the diffraction limit, such as nearfield scanning optical microscopy [1], stimulated-emission-depletion microscopy [2] and photo-activated localisation microscopy [3], and the metamaterial-based superlens [4].

Respectively, in 2009 and in 2011, the ability of nanoscale hemispheres [5] and of microspheres [6] to perform super resolution imaging was also experimentally revealed. Fort this purpose, a dielectric spherical lens is deposited on the object surface to be tested. A magnified image carrying the fine details is then generated [7] in order to be collected by a microscope objective. Both make it possible to observe sub-diffraction-limit features while being label-free, scanning-free and easy-to-implement. Nevertheless, the magnification factor and the resolving power seem to be higher in microsphere-assisted microscopy than in nanolens-based microscopy. Indeed, the microspheres are able to improve the resolving power of an optical microscope by a factor of up to $\times 4$ in air [6] and $\times 6$ in immersion [8] while spherical nanolenses offer a magnification factor of 1.6 in air [5]. Convenient in biological imaging [9-11] and in optical profilometry [12], microsphereassisted microscopy has thus become a powerful technique using a single microsphere or a matrix of microspheres [13].

Nowadays, the phenomenon behind the super resolution is not fully described [14]. It has yet been shown that the photonic jet cannot be interpreted as the point spread function of the imaging system $[15,16]$, despite its promising performance in terms of forming a 
narrow focusing beam [17] for scanning optical imaging [18,19]. The role of coherence in the imaging process has been pointed out where two point objects need to be out-ofphase in order to be resolved [20]. The role of evanescent waves was first experimentally suggested through a decrease in resolution with an increase in the distance microspheresample [21] and more-recently confirmed using semi-immersed $\mathrm{BaTiO}_{3}$ microspheres [22]. To our knowledge, the most advanced explanation has been proposed by Zhou et al. [23] where whispering-gallery modes excited inside the microsphere by high-spatial frequencies from the object make it possible to contribute to a virtual image resolution. However, the numerical demonstration was performed merely by implementing two point sources without indicating the specific contribution of the evanescent waves from the point sources to the image formation in the far field. In 2016, the possible refraction of evanescent waves by microspheres was mathematically demonstrated without any proposition of predictable imaging processing [24]. Therefore, a detailed description of the role of evanescent waves in the microsphere super-resolution imaging process is still expected. In this work, not only the natural coupling of evanescent waves by the glass microspheres, but also their conversion into propagating waves are studied, contributing to the clarification of the physical mechanism of super resolution in microsphere-assisted microscopy.

\section{Method}

To investigate the evanescent wave collection, a rigorous electromagnetic simulation of microsphere assisted microscopy was implemented in the visible range using a finite element method (COMSOL Multiphysics $\left.{ }^{\circledR}\right)$. In the $x-z$ plane, the $2 \mathrm{D}$ model consists of a glass microsphere deposited on a dielectric-air interface at $\mathrm{z}=0 \mu \mathrm{m}$. The transparent microsphere has a refractive index of 1.5 and a diameter $\mathrm{D}$. The surrounding medium of the microsphere is air and the refractive index of the dielectric substrate $n_{s}$ is of 1.5. An oblique excitation plane wave with a wavelength of $\lambda_{0} / n_{S}\left(\lambda_{0}=600 \mathrm{~nm}\right)$ allows the evanescent wave to be generated on the substrate interface using total internal reflection. The incident angle $\theta$ is of $1 \mathrm{rad}$ which is larger than the critical angle $\left(\sin \theta_{c}=1 / n_{s}\right)$. The incident coherent excitation wave has a unit electric field, giving a radiated power density $P_{0}=\epsilon c|E|^{2} / 2$ with $\epsilon$ the permittivity of the dielectric substrate. The evanescent wave thus propagates along the x-axis and exponentially decays along the z-axis. According to the complex Snell-Descartes's law, the wave vector components of the evanescent wave in air can be written as:

$$
\vec{K}= \begin{cases}k_{0} n_{s} \sin \theta & \mathrm{x} \text { axis } \\ i k_{0} \sqrt{n_{s}^{2} \sin ^{2} \theta-1} & \mathrm{z} \text { axis }\end{cases}
$$

where $k_{0}$ is the wavenumber of the excitation plane wave in free-space. The imaginary unit $i$ satisfies the relation $i^{2}=-1$. Finally, perfectly matched layers surround the calculation area [25].

\section{Results}

Figure 1 shows the natural collection of the evanescent wave travelling along the interface by a 3- $\mu \mathrm{m}$-diameter glass microsphere. The checked pattern in the dielectric substrate, in Figure 1a, results from interferences between the excitation plane wave and the wave reflected by the interface. Whispering gallery modes (WGMs) can be observed inside the microsphere. The highest peak amplitude of the WGM, equalling $3.7 \mathrm{~V} / \mathrm{m}$, occurs within the microsphere at a radius of around $1.4 \mu \mathrm{m}$. 


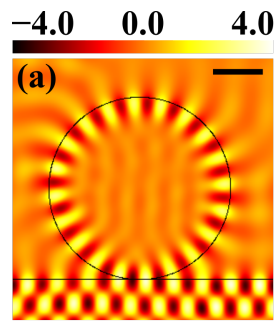

$\psi=\pi$

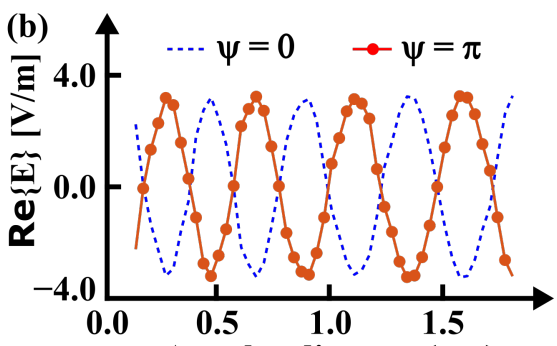

Angular distance $(\mu \mathrm{m})$

Figure 1. Collection of an evanescent wave by a glass microsphere (see Visualization 1 in Supplementary Materials). (a) Real part of the electric field distributions when the initial phase $\psi$ of the excitation wave is of $\pi$. Black-scale bars represent $1 \mu \mathrm{m}$. (b) Radial section profiles at a radius of $1.4 \mu \mathrm{m}$ of (a) as a function of the angular position when $\psi$ equals $0 \mathrm{rad}$ and $\pi \mathrm{rad} . \lambda_{0}=600 \mathrm{~nm}$, $\theta=1 \mathrm{rad}, n_{s}=1.5, \mathrm{D}=3 \mu \mathrm{m}$.

In order to emphasise the WGM generation from the evanescent wave and to confirm the coupling of the near-field wave by the microsphere, the initial phasor $\psi$ of the excitation plane wave is swept from 0 to $2 \pi$. Increasing the offset $\psi$ leads to a rotation of the WGMs (see Visualization 1 in Supplementary Materials). The rotational angle of the WGM patterns equals the initial phasor $\psi$. Indeed, as shown in Figure $1 \mathrm{~b}$, when $\psi$ is of $0 \mathrm{rad}$ and $\pi \mathrm{rad}$, the angular sections of the electric field inside the sphere at a radius of $1.4 \mu \mathrm{m}$ are also phase-shifted by $\pi$. Along the microsphere rim, the period of the WGM electric field equals $475 \mathrm{~nm}$, i.e., the sub-wavelength period of the evanescent wave $\lambda_{0} /\left(n_{s} \times \sin \theta\right)$. At the radial position of $1.4 \mu \mathrm{m}$, the period of the WGM is smaller (around $445 \mathrm{~nm}$ ). The peak amplitudes are, for their parts, not affected by the phase delay $\psi$ (e.g., the highest peak amplitude of the WGM remains at around $3.7 \mathrm{~V} / \mathrm{m}$ ).

Figure 2 shows the ability of the 3 - $\mu$ m-diameter microsphere to collect the evanescent waves according to its axial position above the interface.

$-4.0$

$\mathbf{0 . 0}$

4.0

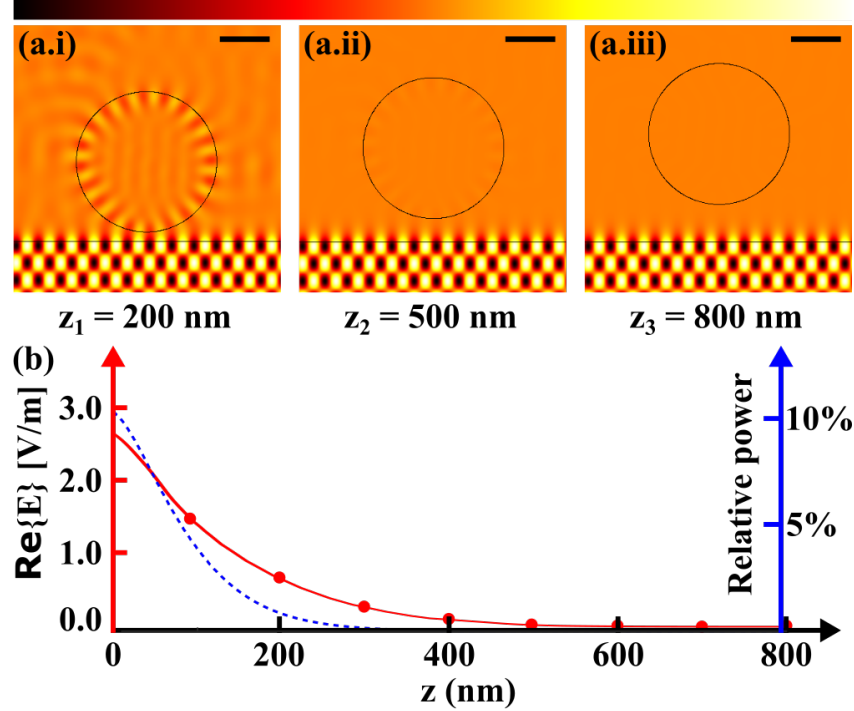

Figure 2. Collection efficiency of an evanescent wave by a glass microsphere at an axial gap with the interface. (a.i), (a.ii) and (a.iii) Real parts of the electric field distributions when the microsphere is positioned at a distance of $200 \mathrm{~nm}, 500 \mathrm{~nm}$ and $800 \mathrm{~nm}$ from the interface, respectively. Black-scale bars represent $1 \mu \mathrm{m}$. (b) Real-part peak amplitude of the WGM electric field along the microsphere rim and relative power transmitted by the microsphere in the far field as a function of the $\mathrm{z}$ distance. $\lambda_{0}=600 \mathrm{~nm}, \theta=1 \mathrm{rad}, \psi=0 \mathrm{rad}, n_{\mathrm{s}}=1.5, \mathrm{D}=3 \mu \mathrm{m}$.

Increasing the air gap allows not only to attest the evanescent wave coupling by the microsphere, but also to predict the longest acceptable distance, thus making it possible 
to perform contact-less measurements in microsphere-assisted microscopy. Indeed, the electric-field amplitude maximum of the WGM exponentially decreases according to the axial distance. In this case, the coupling efficiency of the evanescent wave by the microsphere appears to be low beyond a distance $z=400 \mathrm{~nm}$. In Figure $2 \mathrm{~b}$, the far-field relative radiated power was estimated by considering the incident power $P_{0}$ through the radius of the estimated interaction area [21], i.e., $\sqrt{2 \lambda_{0} h}$ with $h$, the penetration depth measuring around $125 \mathrm{~nm}$ in this configuration $\left(1 /\left|k_{z}\right|\right.$, see Equation (1)). The optical power emanating from the top of the microsphere is not null and decreases as distance increases (e.g., $0.9 \% P_{0}$ at $\mathrm{z}=200 \mathrm{~nm}$ and $0.1 \% P_{0}$ at $\mathrm{z}=400 \mathrm{~nm}$ ). In this case, the distance of $400 \mathrm{~nm}$ can be seen as the depth of field of the imaging technique.

A slight variation of the microsphere diameter obviously leads to modifications of the excited WGMs. Figure 3a highlights the peak amplitude fluctuations of the WGMs along the microsphere rim by varying the diameter from $3.0 \mu \mathrm{m}$ to $3.3 \mu \mathrm{m}$. The microsphere is in contact with the interface. The amplitude of the electric field progresses following a periodic wave as a function of the diameter. In this case, periodic resonances occur when the microsphere diameter measures $3.03 \mu \mathrm{m}$ and $3.17 \mu \mathrm{m}$ which correspond to multiples of $475.3 \mathrm{~nm} / \pi$. As a matter of fact, the multiple is the number of radial periods of the WGM, i.e., 20 and 21 periods.
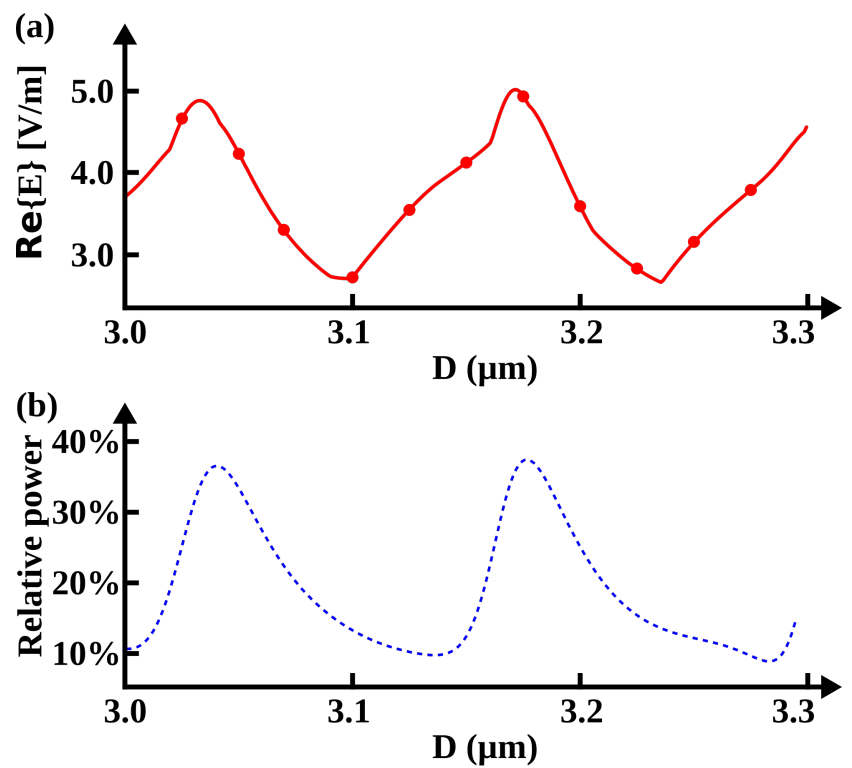

Figure 3. Influence of the microsphere diameter (a) on the peak amplitude of the real part of the electric field along the microsphere rim and (b) on the transmitted power. $\lambda_{0}=600 \mathrm{~nm}, \theta=1 \mathrm{rad}$, $\psi=0 \mathrm{rad}, n_{s}=1.5$.

Close resonances may appear but are not required to benefit from the evanescent wave coupling effect. As shown in Figure 3a, the resonances only increase the coupling efficiency. In addition, simulations were made (in the contact mode) in order to quantify the difference of the power transmitted in the far field by the microsphere for a resonant and a non-resonant WGM (see Figure $3 \mathrm{~b}$ ). $\mathrm{n}$ the far field, the transmitted power is increased by a factor of nearly $4\left(\right.$ at $\mathrm{D}=3.14 \mu \mathrm{m}, 9.8 \% P_{0}$, and at $\left.\mathrm{D}=3.18 \mu \mathrm{m}, 37.5 \% P_{0}\right)$. It can be observed that far field power peaks occur when the WGM electric field is maximum (at $\mathrm{D}=3.03 \mu \mathrm{m}$ and $\mathrm{D}=3.17 \mu \mathrm{m}$ ). For the minima, this correlation is not found, indicating that the WGM generation is not the only phenomenon behind far field propagation.

Without the microsphere, the evanescent wave would propagate along the dielectricair interface on the x-axis, i.e., a microscope objective alone would not be able to collect the high-spatial-frequency optical signal. However, by introducing the glass microsphere, the evanescent wave is not only coupled, but also appears to be propagated in the far field. Propagating-spherical waves emanate from the microsphere, demonstrating its ability to 
convert a near-field wave into a far-field wave. In experiments, these propagating waves are collected by a microscope objective. Figure 4 exposes this phenomenon in air when the period of the evanescent wave is $475 \mathrm{~nm}, 355 \mathrm{~nm}$ and $240 \mathrm{~nm}$. For this purpose, the refractive index $n_{s}$ of the dielectric substrate is varied (such refractive indices cannot be achieved in reality). Increasing the refractive index $n_{s}$ also makes the periods of WGMs narrower. The optical power transmitted by the microsphere in the far field is of $10.9 \% P_{0}$, $0.6 \% P_{0}$ and $7.2 \times 10^{-5 \%} P_{0}$ when $n_{s}$ is of $1.5,2.0$ and 3.0, respectively. In Figure $4 \mathrm{c}, \mathrm{d}$, the amplitude and the intensity ranges are saturated in order to emphasise the relatively-low amplitude values of the propagating wave.

Thus, the ability of the microsphere to collect the highest spatial frequencies appears to be more complex. Recently, Lai et al. have experimentally highlighted that using a larger microsphere would lead to an increased efficiency of the transmitted intensity, but would reduce the resolving power [26]. In addition, a broadband light source usually illuminates the microsphere in experiments (instead of a coherent illumination). Due to the equivalence between the sphere diameter increase and the wavelength decrease in electromagnetism, Figure 3 shows that several nearby resonances will occur together using a broadband light source. Thus, each spectral component (or wavelength-dependent evanescent wave) would contribute to the conversion efficiency by the microsphere with more-or-less ability, yielding an increase in propagating-wave intensities. Moreover, to improve the signal-to-noise ratio of the imaging contrast, plasmonic-based illumination has been suggested [27]. Finally, we can notice that increasing the spatial frequency of the evanescent wave leads to a decrease in the attenuation distance of the near-field wave and, thus, to a decrease in the axial distance along which evanescent waves can be collected (e.g., $400 \mathrm{~nm}$ when $n_{s}=1.5$ ).

$\begin{array}{lll}-4.0 & 0.0 & 4.0\end{array}$

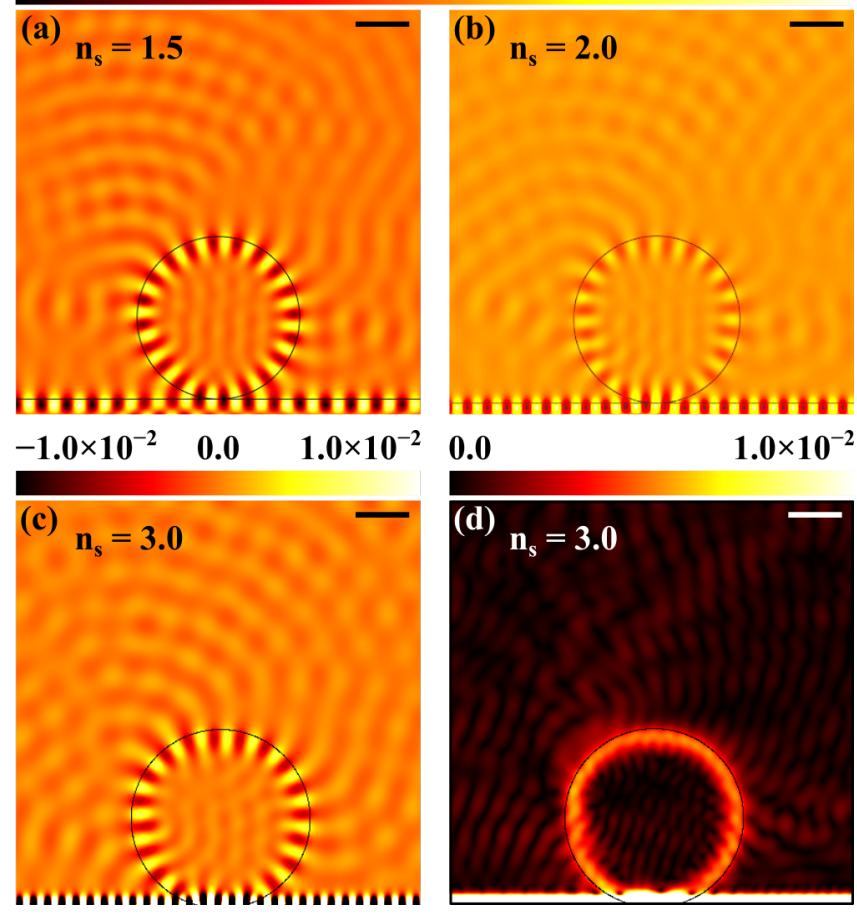

Figure 4. Conversion of an evanescent wave in a propagative wave by a glass microsphere. Real parts of the electric field distributions when (a) $n_{S}=1.5$, (b) $n_{S}=2.0$ and (c) $n_{S}=3.0$. (d) Absolute value of the complex electric field distribution when $n_{s}=3.0$. Scale bars represent $1 \mu \mathrm{m} . \lambda_{0}=600 \mathrm{~nm}$, $\theta=1 \mathrm{rad}, \psi=0 \mathrm{rad}, \mathrm{D}=3 \mu \mathrm{m}$.

Figure 4 also shows that the outgoing propagating waves do not come from everywhere on the microsphere, but from specific points on its surface. This is due to the fact 
that the sphere interaction with the surface breaks the axial symmetry of the WGM [28]. This observation is important. More than a simple power collection (as in NSOM), this demonstrates the necessary relation between the spatial frequency and initial phasor $\psi$ coming from the object plane and the spatial emitting position, a prerequisite for achieving full-field imaging.

In experiments, immersion of high-refractive-index microspheres may be required such as in biological imaging $[8,29]$ or may offer the possibility of manipulating microspheres above the sample [30]. Working in immersion increases not only the resolving power, but also the imaging contrast. Indeed, a more efficient ability of microspheres in immersion to collect high-frequency waves occurs in immersion, enhancing the experimental conditions and performance. The gap distance would appear to be reduced in immersion.

\section{Conclusions}

The phenomenon behind super resolution in microsphere-assisted microscopy has been investigated. Based on a finite element method, rigorous electromagnetic simulations in the visible range have shown not only the natural collection of evanescent waves by dielectric microspheres through WGMs, but also their conversion into propagating waves. The near-field high-spatial frequencies of an object are naturally coupled without requiring resonances contributing to the explanation of the sub-diffraction-limit imaging ability of glass microspheres. Furthermore, it aims at proposing a comprehensive physical imaging mechanism. An analogy with the principle of near-field scanning optical microscopy can thus be made with enhancements such as the wide-field measurement. In future studies, a complete description of the imaging process using will be implemented in order to quantify the resolving power according to geometrical and optical parameters. Moreover, simulations through microspheres having a diameter of larger than $3 \mu \mathrm{m}$ (as is often the case in experiments) and the influence of WGMs on the spectrum of the broadband light source will be considered.

Supplementary Materials: The following are available online at www.mdpi.com/xxx/s1.

Author Contributions: Methodology, R.B. and S.L.; software, R.B. and S.L.; validation, S.P. and S.L.; formal analysis, R.B.; investigation, R.B., S.P. and S.L.; resources, S.L.; data curation, R.B. and S.P.; writing—original draft preparation, R.B.; writing—review and editing, S.P., A.D., P.M., N.-E.D. and S.L.; visualization, R.B. and S.P.; supervision, A.D., P.M., N.-E.D. and S.L.; project administration, N.-E.D. and S.L.; funding acquisition, N.-E.D. and S.L. All authors have read and agreed to the published version of the manuscript.

Funding: SATT Conectus Alsace; PHC TASSILI Cooperation Program; University of Strasbourg.

Institutional Review Board Statement: Not applicable.

Informed Consent Statement: Not applicable.

Acknowledgments: The authors thank Amandine Elchinger for her useful comments in the editing of the manuscript.

Conflicts of Interest: The authors declare no conflict of interest.

\section{References}

1. Pohl, D.W.; Courjon, D. Near Field Optics; Springer: Amsterdam, The Netherlands, 1993.

2. Hell, S.W.; Wichmann, J. Breaking the diffraction resolution limit by stimulated emission: Stimulated-emission-depletion fluorescence microscopy. Opt. Lett. 1994, 19, 780-782. [CrossRef]

3. Betzig, E.; Patterson, G.H.; Sougrat, R.; Lindwasser, O.W.; Olenych, S.; Bonifacino, J.S.; Davidson, M.W.; Lippincott-Schwartz, J.; Hess, H.F. Imaging intracellular fluorescent proteins at nanometer resolution. Science 2006, 313, 1642-1645. [CrossRef]

4. Smith, D.R.; Schurig, D.; Rosenbluth, M.; Schultz, S.; Ramakrishna, S.A.; Pendry, J.B. Limitations on subdiffraction imaging with a negative refractive index slab. Appl. Phys. Lett. 2003, 82, 1506-1508. [CrossRef]

5. $\quad$ Lee, J.Y.; Hong, B.H.; Kim, W.Y.; Min, S.K.; Kim, Y.; Jouravlev, M.V.; Bose, R.; Kim, K.S.; Hwang, I.-C.; Kaufman, L.J.; Wong, C.W.; Kim, P.; Kim, K.S. Near-field focusing and magnification through self-assembled nanoscale spherical lenses. Nature 2009, 460, 498-501. [CrossRef] 
6. Wang, Z.; Guo, W.; Li, L.; Luk'yanchuk, B.; Khan, A.; Liu, Z.; Chen, Z.; Hong, M. Optical virtual imaging at 50 nm lateral resolution with a white-light nanoscope. Nat. Commun. 2011, 2, 218. [CrossRef] [PubMed]

7. Perrin, S.; Li, H.; Lecler, S.; Montgomery, P. Unconventional magnification behaviour in microsphere-assisted microscopy. Opt. Laser Technol. 2019, 114, 40-43. [CrossRef]

8. Darafsheh, A. Influence of the background medium on imaging performance of microsphere-assisted super-resolution microscopy. Opt. Lett. 2017, 42, 735-738. [CrossRef] [PubMed]

9. Li, L.; Guo, W.; Yan, Y.; Lee, S.; Wang, T. Label-free super-resolution imaging of adenoviruses by submerged microsphere optical nanoscopy. Light Sci. Appl. 2013, 2, e104. [CrossRef]

10. Wang, F.; Liu, L.; Yu, H.; Wen, Y.; Yu, P.; Liu, Z.; Wang, Y.; Li, W.J. Scanning superlens microscopy for non-invasive large field-of-view visible light nanoscale imaging. Nat. Commun. 2016, 7, 13748. [CrossRef] [PubMed]

11. Perrin, S.; Li, H.; Badu, K.; Comparon, T.; Quaranta, G.; Messaddeq, N.; Lemercier, N.; Montgomery, P.; Vonesch, J.-L.; Lecler, S. Transmission microsphere-assisted dark-field microscopy. Phys. Status Solidi RRL 2019, 13, 1800445. [CrossRef]

12. Perrin, S.; Donie, Y.J.; Montgomery, P.; Gomard, G.; Lecler, S. Compensated Microsphere-Assisted Interference Microscopy. Phys. Rev. Appl. 2020, 13, 014068. [CrossRef]

13. Allen, K.W.; Farahi, N.; Li, Y.; Limberopoulos, N.I.; Walker, D.E.; Urbas, A.M.; Astratov, V.N. Overcoming the diffraction limit of imaging nanoplasmonic arrays by microspheres and microfibers. Opt. Express 2015, 23, 24484-24496. [CrossRef] [PubMed]

14. Duan, Y.; Barbastathis, G.; Zhang, B. Classical imaging theory of a microlens with super-resolution. Opt. Lett. 2013, 38, 2988-2990. [CrossRef]

15. Maslov, A.; Astratov, V. Resolution and reciprocity in microspherical nanoscopy: Point-spread function versus photonic nanojets. Phys. Rev. Appl. 2019, 11, 064004. [CrossRef]

16. Lecler, S.; Perrin, S.; Leong-Hoi, A.; Montgomery, P. Photonic jet lens. Sci. Rep. 2019, 9, 4725. [CrossRef] [PubMed]

17. Darafsheh, A. Photonic nanojets and their applications. J. Phys. Photonics 2021, 3, 022001. [CrossRef]

18. Yi, K.J.; Wang, H.; Lu, Y.F.; Yang, Z.Y. Enhanced Raman scattering by self-assembled silica spherical microparticles. J. Appl. Phys. 2007, 101, 063528. [CrossRef]

19. Tehrani, K.F.; Darafsheh, A.; Phang, S.; Mortensen, L.J. Resolution enhancement of 2-photon microscopy using high-refractive index microspheres. Proc. SPIE 2018, 10498, 1049833.

20. Kassamakov, I.; Lecler, S.; Nolvi, A.; Leong-Hoi, A.; Montgomery, P.; Haeggstrom, E. 3D super-resolution optical profiling using microsphere enhanced Mirau interferometry. Sci. Rep. 2017, 7, 3683. [CrossRef] [PubMed]

21. Lin, Q.; Wang, D.; Wang, Y.; Rong, L.; Zhao, J.; Guo, S.; Wang, M. Super-resolution imaging by microsphere-assisted optical microscopy. Opt. Quantum Electron. 2016, 48, 557. [CrossRef]

22. Yang, S.; Ye, Y.-H.; Shi, Q.; Zhang, J. Converting Evanescent Waves into Propagating Waves: The Super-Resolution Mechanism in Microsphere-Assisted Microscopy. J. Phys. Chem. A C 2020, 124, 25951-25956. [CrossRef]

23. Zhou, S.; Deng, Y.; Zhou, W.; Yu, M.; Urbach, H.P.; Wu, Y. Effects of whispering gallery mode in microsphere super-resolution imaging. 2017, 123, 236 [CrossRef]

24. Ben-Aryeh, Y. Increase of resolution by use of microspheres related to complex Snell's law. JOSA A 2016, 33, 2284-2288. [CrossRef] [PubMed]

25. Berenger, J.-P. A perfectly matched layer for the absorption of electromagnetic waves. J. Comput. Phys. 1994, 114, 185-200. [CrossRef]

26. Lai, H.S.S.; Wang, F.; Li, Y.; Jia, B.; Liu, L.; Li, W.J. Super-resolution real imaging in microsphere-assisted microscopy. PLoS ONE 2016, 11, e0165194. [CrossRef]

27. Astratov, V.N.; Brettin, A.; Abolmaali, F.; Poffo, L.; Maslov, A.V. Plasmonics and Super resolution in Microspherical Nanoscopy. In Proceedings of the 20th International Conference on Transparent Optical Networks (ICTON), Bucharest, Romania, 1-5 July 2018; pp. 1-4.

28. Mollaei, M.; Simovski, C. Dual-metasurface superlens: A comprehensive study. Phys. Rev. B 2019, 100, 205426. [CrossRef]

29. Darafsheh, A.; Walsh, G.F.; Negro, L.D.; Astratov, V.N. Optical super-resolution by high-index liquid-immersed microspheres. Appl. Phys. Lett. 2012, 101, 141128. [CrossRef]

30. Zhang, T.; Li, P.; Yu, H.; Wang, F.; Wang, X.; Yang, T.; Yang, W.; Li, W.J.; Wang, Y.; Liu, L. Fabrication of flexible microlens arrays for parallel super-resolution imaging. Appl. Surf. Sci. 2020, 504, 144375. [CrossRef] 